\title{
Vacuous Jaw Movements and Feeding Deficits in Rats with Ventrolateral Striatal Dopamine Depletion: Possible Relation to Parkinsonian Symptoms
}

\author{
Gregory A. Jicha and John D. Salamone \\ Department of Psychology, University of Connecticut, Storrs, Connecticut 06269-1020
}

\begin{abstract}
A series of experiments examined the effects of regional dopamine depletions produced by intrastriatal injections of 6-hydroxydopamine on voluntary and involuntary movements in rats. Depletion of dopamine in the ventrolateral striatum produced a substantial decrease in food intake, from which the animals recovered. Rats with dopamine depletions in anteroventromedial or dorsolateral striatum did not have significant feeding deficits. Rats with ventrolateral dopamine depletions showed no deficits in locomotor activity or rearing behavior; however, depletions of dopamine in dorsolateral striatum significantly reduced rearing. Vacuous jaw movements that resemble chewing were produced by dopamine depletion in the ventrolateral striatum, but not the anteroventromedial or dorsolateral striatum. Systemic administration of haloperidol $(0.4 \mathrm{mg} / \mathrm{kg})$ increased vacuous chewing responses in dopamine-depleted and control rats. Thus, vacuous chewing responses can result from reduced functional activity of striatal dopamine, and these responses share some characteristics with human parkinsonian symptoms. In addition, these data support the notion that the neostriatum is functionally heterogeneous and that the ventrolateral region is particularly important for oral motor control.
\end{abstract}

Striatal dopamine (DA) is involved in various motor and sensorimotor functions. Extensive depletion of striatal DA in rats produces aphagia, adipsia, and akinesia and reduces responsiveness to various sensory stimuli (Ungerstedt, 1971; Marshall et al., 1974; Stricker and Zigmond, 1976), yet despite the considerable volume of research in this area, it is not clear how DA in spccific striatal subrcgions is involved in different aspects of motor control. Considerable evidence indicates that the striatum of the rat, like the caudate and putamen in primates, is functionally heterogeneous and that the lateral striatum is particularly involved in aspects of motor and sensorimotor function. The decreases in food intake and feeding efficiency produced by forebrain DA depletion were correlated with depletion of DA in the lateral, but not the medial, striatum (Salamone et al., 1990c). Depletions of DA in lateral striatum produced by intrastriatal injections of 6-hydroxydopamine (6-OHDA) decreased responsiveness to cutaneous stimuli (Dunnett and Iver-

Received Apr. 12, 1991; revised May 29, 1991; accepted July 3, 1991.

This research was supported by University of Connecticut Research Foundation Grant 35-903 and National Science Foundation Grant BNS-9009613.

Correspondence should be addressed to John D. Salamone, Department of Psychology, 406 Babbidge Road, University of Connecticut, Storrs, CT 062691020.

Copyright (C) 1991 Society for Neuroscience $0270-6474 / 91 / 113822-08 \$ 05.00 / 0$ sen, 1982; Fairley and Marshall, 1987) and have been shown to impair forelimb reaching (Evenden and Robbins, 1984; Sabol et al., 1985; Whishaw et al., 1986). Ibotenic acid lesions of the lateral striatum produced deficits in food handling and biting (Pisa, 1988a,b). It has been suggested that the lateral striatum of the rat is somatotopically organized (Pisa, 1988a; Pisa and Schranz, 1988) and that the ventrolateral striatum is involved in oral and forelimb motor control (Kelley et al., 1988, 1989; Pisa and Schranz, 1988; Salamone et al., 1990a).

Another important consideration is the extent to which depletion of DA in rats can be used to model symptoms of human parkinsonism. The decrease in locomotor activity produced by DA depletion in rats is reversible with the antiparkinsonian drugs L-dopa (Uretsky and Schoenfeld, 1971) and atropine (Schallert et al., 1978). In addition, DA-depleted rats that are akinetic still can exhibit motor responses to intense stimuli (Marshall et al., 1976; Keefe et al., 1989), which is similar to the paradoxical kinesia shown by parkinsonian patients (Schwab and Zieper, 1965). These data indicate that the akinesia shown by DA-depleted rats has some of the characteristics of human parkinsonism. However, another important symptom of Parkinson's disease is involuntary movements, such as tremor. Thus far, there have been very few reports of tremor in DA-depleted rats (e.g., Buonamici et al., 1986).

Oral movements have been observed in rats treated with DA antagonists (Iversen et al., 1980; Rupniak et al., 1983, 1985, 1986; Johansson et al., 1986; Ellison et al., 1987; Ellison and See, 1989; Salamone et al., 1990b). In addition, similar oral movements are produced by cholinomimetic drugs (Rupniak et al., 1983; Salamone et al., 1986, 1990a,b). The most prominent behavior is "vacuous chewing," a rapid jaw movement not directed at any particular stimulus. These movements tend to occur in rapid, repetitive bursts in which the lower jaw oscillates vertically (Salamone et al., 1986, 1990a). There is considerable debate about the relation between vacuous chewing in rats and human movement disorders (Rupniak et al., 1986; Waddington and Molloy, 1987; Ellison and See, 1989; Kelley et al., 1989; Stoessl et al., 1989; Salamone et al., 1990a; Waddington, 1990), and some researchers have suggested that vacuous chewing is related to tardive dyskinesia (Ellison and See, 1989) or acute dystonia (Rupniak et al., 1986). However, Salamone et al. (1990a) observed that vacuous chewing in rats shares characteristics with human parkinsonian symptoms. Vacuous chewing can be induced by acute or subchronic administration of DA antagonists (Glassman and Glassman, 1980; Rupniak et al., 1985; Salamone et al., 1990b). Both vacuous chewing and parkinsonism can be produced or exacerbated by cholinomimetic drugs (Duvoisin, 
1967; Rupniak et al., 1983, 1985; Harbaugh et al., 1984; Noring et al., 1984; Salamone et al., 1986). Because idiopathic Parkinson's disease is related to striatal DA depletion, it would be useful to determine if striatal DA depletion in rats can induce vacuous chewing movements.

The present studies were undertaken to determine if localized depletion of DA by intrastriatal injections of 6-OHDA could impair motor function and induce vacuous chewing movements. Injections of cholinomimetic drugs into the ventrolateral striatum (VLS) were shown to induce vacuous chewing (Kelley et al., 1989; Salamone et al., 1990a), and an initial report from our laboratory has indicated that DA depletion in the VLS also produces vacuous chewing (Salamone et al., 1990b). In the present series of studies, three striatal sites were studied: the VLS, the anteroventromedial striatum (AVMS), and the dorsolateral striatum (DLS). Behavioral measures included locomotor activity, food intake, vacuous chewing movements, and rearing. In the final phase of the experiment, all rats received injections of haloperidol (HP) to study the combined effects of DA depletion and pharmacological blockade.

\section{Materials and Methods}

Animals. The subjects were male Sprague-Dawley rats obtained from Blue Spruce Farms (Altamont, NY) and weighed 350-450 gm. The colony in which they were housed provided a stable room temperature and a $12 \mathrm{hr}$ light/ $12 \mathrm{hr}$ dark cycle. All testing was performed 4-8 hr after the onset of the light cycle. The animals were maintained with food and water ad libitum. Rats with reduced food intake after surgery were fed sweetened wet mash for brief periods (1-2 hr) in order to maintain normal weights.

Drugs. HP was ohtained from Sigma Chemical Co. and was dissolved in $0.3 \%$ tartaric acid vehicle. For control injections, rats received injection of tartaric acid vehicle alone.

Surgery. 6-OHDA HBr was dissolved in $0.1 \%$ ascorbic acid solutions to prevent oxidation. Prior to surgery, all animals were placed under pentabarbital anesthesia. Bilateral injections of either 6-OHDA or ascorbic acid solutions were delivered via 30 ga tubing that was connected to a Hamilton microsyringe with polyethylene tubing. Flow rate for the injections was $0.75 \mu \mathrm{l} / \mathrm{min}$, which was controlled by a Harvard Apparatus syringe pump. Injection volumes were $2.5 \mu \mathrm{l}$ per hemisphere with the 6-OHDA solution containing $4.0 \mu \mathrm{g} / \mu \mathrm{l}$ of the free base $(10 \mu \mathrm{g}$ of 6-OHDA per side). Control animals were injected with $2.5 \mu \mathrm{l}$ per hemisphere of the ascorbic acid vehicle. Stereotaxic placements of the injector tips were at the following coordinates: VLS: AP $1.6 \mathrm{~mm}, \mathrm{~L} \pm 4.0 \mathrm{~mm}$, V $7.2 \mathrm{~mm}$ from the skull; DLS: AP $1.6 \mathrm{~mm}, \mathrm{~L} \pm 3.0 \mathrm{~mm}, \mathrm{~V} 4.2 \mathrm{~mm}$ from the skull; AVMS: AP $2.2 \mathrm{~mm}, \mathrm{~L} \pm 2.0 \mathrm{~mm}, \mathrm{~V} 6.5 \mathrm{~mm}$ from the skull (incisor bar was raised $5.0 \mathrm{~mm}$ above interaural line). Rats received intrastriatal injections of 6-OHDA into either the VLS $(n=9)$, the $\operatorname{AVMS}(n=9)$, or the DLS $(n=9)$, and rats in the control group were injected with ascorbic acid vehicle $(n=9,3$ per site).

Behavioral observations. Body weight and daily food intake (correcting for spillage) was recorded for the first $7 \mathrm{~d}$ after surgery. In any rats that received wet mash, the mash was given in the $1-2 \mathrm{hr}$ period in the afternoon during which the lab chow was being weighed, and the mash was taken away when weighed amounts of lab chow were placed back in the home cage. On the third day after surgery, rats were tested for locomotor activity in an automated chamber constructed in the laboratory. The chamber was a Plexiglas box $(25 \times 25 \times 25 \mathrm{~cm})$ with two rectangular floor panels, and movements of the rats that moved the floor panels were registered by microswitches and electromechanical counters. Activity counts were recorded in five $4 \mathrm{~min}$ intervals for a period of $20 \mathrm{~min}$.

Observation chambers for recording vacuous chewing responses consisted of $28 \times 28 \times 28 \mathrm{~cm}$ Plexiglas boxes resting on a wire mesh floor. Prior to surgery, all rats were habituated to the oral movement observation chamber in two $30 \mathrm{~min}$ sessions. Vacuous chewing was defined as a rapid vertical deflection of the lower jaw that resembled normal chewing but was not directed at any stimulus. Each individual jaw movement was recorded on an electromechanical counter by an ob- server who was unaware of the experimental condition. Animals were observed on days 5,7 , and 9 after surgery in 10 min sessions. Rearing responses also were recorded in these observation periods. Behavioral observations were conducted prior to the weighing of food and the presentation of mash on that day.

Effects of haloperidol. The animals were administered $0.4 \mathrm{mg} / \mathrm{kg} \mathrm{HP}$ or $1.0 \mathrm{ml} / \mathrm{kg}$ tartaric acid vehicle on days 13 and 18 after surgery, with all rats receiving both treatments in a randomized order. The effects of drug treatment on vacuous chewing and rearing responses were assessed by an observer who was blind to the experimental condition. All rats were observed in $10 \mathrm{~min}$ sessions $50-60 \mathrm{~min}$ after injection.

HPLC assays for dopamine. Rats were assayed to assess the effects of 6-OHDA on tissue levels of DA. After the termination of the experiment, rats were decapitated, and the brains were removed immediately and placed in ice water. The brains were frozen and cut into 0.7 -mm-thick coronal sections using a freezing microtome. Hollow stainless-steel tubing (16 ga) was used to cut cylindrical sections from the nucleus accumbens, AVMS, VLS, and DLS for assays. These samples were placed in $200 \mu \mathrm{l}$ of $0.1 \mathrm{~N}$ perchloric acid solution and homogenized. The homogenized sample was centrifuged, and the supernatant was used for neurochemical analysis using an HPLC system (Waters pump, reverse-phase column, Coulochem electrochemical detector, chart recorder). Standards of DA were assayed before, during, and after the samples to calculate nanogram quantities of DA per milligram of wet tissue.

Statistical analysis. Data from this experiment were analyzed using a factorial ANOVA with repeated measures. Newman-Keuls multiple comparisons were used for post hoc analysis of data. Data on DA levels were assessed by simple ANOVA within each brain region assayed. Pearson product-moment correlation coefficients were also used to assess relations between feeding (total food intake), vacuous chewing (total number of chews on days 5, 7 and 9), rearing (total rears), locomotor activity (total activity counts), and neurochemical data (DA levels in each region).

\section{Results}

\section{Food intake}

Daily food intake was affected by injections of 6-OHDA (Fig. 1). ANOVA showed that there was a significant treatment effect $[F(3,32)=11.5 ; p<0.001]$, a significant effect of observation days $[F(6,192)=34.9 ; p<0.001]$, and a significant group $\times$ day interaction effect $[F(18,192)=2.2 ; p<0.01]$. Post hoc comparisons showed that rats with VLS DA depletion differed significantly from control rats on days $1-4$, but rats with AVMS and DLS placements did not differ from control rats. Observations of the rats during feeding indicated that rats with VLS DA depletions attempted to eat but had difficulty manipulating and biting large dry food pellets. Even the most severely impaired rat with VLS DA depletion in our study was capable of maintaining its body weight by consumption of wet mash, and none of the rats required tube feeding.

\section{Locomotor activity}

Figure 2 shows the results of the locomotor activity test. There was no overall difference in locomotor activity counts between groups $[F(3,32)=0.86, \mathrm{NS}]$, but there was a significant effect of time interval $[F(4,128)=34.5 ; p<0.001]$, and a significant group $\times$ interval interaction $[F(12,128)=2.5 ; p<0.01]$. Post hoc analyses indicated that there was a significant increase in activity in rats with VLS injections of 6-OHDA relative to control rats in the first 4 min period.

\section{Vacuous chewing and rearing}

VLS injections of 6-OHDA produced an increase in vacuous chewing as compared to control animals (Fig. 3). Factorial ANOVA with repeated measures showed a significant effect of trcatmcnt $[F(3,32=33.1 ; p<0.001]$ and a significant effect of observation days $[F(2,64=4.5 ; p<0.05]$ but no significant 


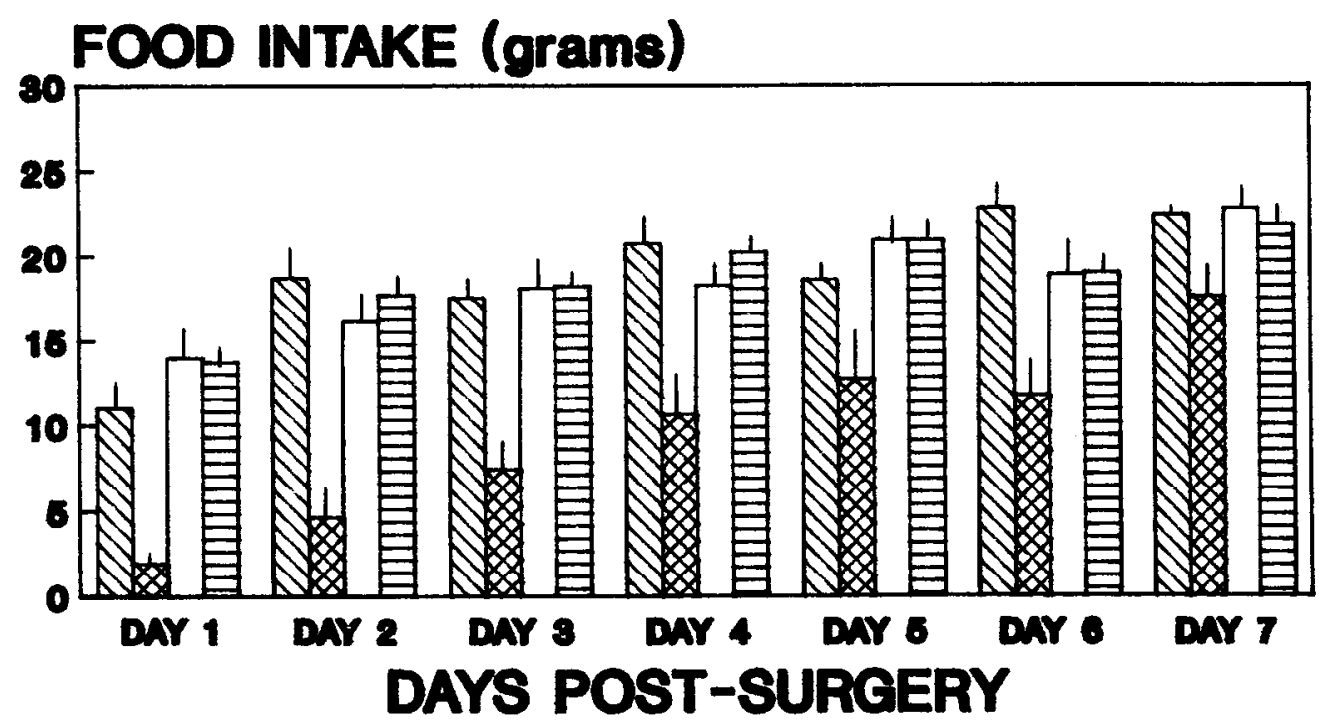

Figure 1. Mean ( \pm SEM) daily food intake for the first $7 \mathrm{~d}$ after surgery.

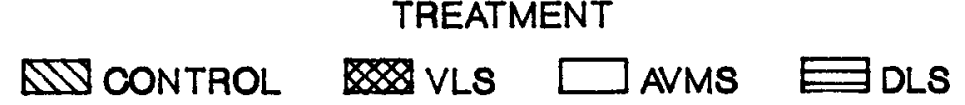

treatment $\times$ day interaction $[F(6,64)=0.6, \mathrm{NS}]$. Post hoc tests showed that animals with VLS DA depletions differed significantly from controls on all test days, whereas animals with AVMS and DLS placements did not. Forelimb and cheek tremors also were observed in many of the animals that had received 6-OHDA injections in the VLS, and it was noted that rats showing higher levels of vacuous chewing were most often the same rats that exhibited other symptoms of tremor. Within large bursts of chews, it was estimated that the local frequency of chewing responses was approximately $4 \mathrm{~Hz}$.

The rearing data for all observations are shown in Figure 4. The results of ANOVA revealed a significant treatment effect $[F(3,32)=8.8 ; p<0.01]$, but no effect of observation days
$[F(2,64)=1.0, \mathrm{NS}]$ and no significant group $\times$ day interaction $[F(6,64)=0.2$, NS $]$. Post hoc analysis demonstrated that the rats with DLS placements showed a significant suppression of rearing, whereas the other two placement groups did not.

\section{Effects of haloperidol}

Systemic administration of HP increased vacuous chewing in all four experimental groups (Fig. 5). ANOVA results showed that there was a significant effect of experimental group on vacuous chewing $[F(3,32)=7.1 ; p<0.01]$, and there was a significant effect of HP administration on chewing $[F(1,32)=69.9$; $p<0.001]$. There was no significant group $\times$ drug interaction effect $[F(3,32)=2.3$, NS $]$. The combination of VLS DA deple-
Figure 2. Mean ( $\pm \mathrm{SEM})$ locomotor activity counts for all groups of rats, obtained in five 4 min periods.

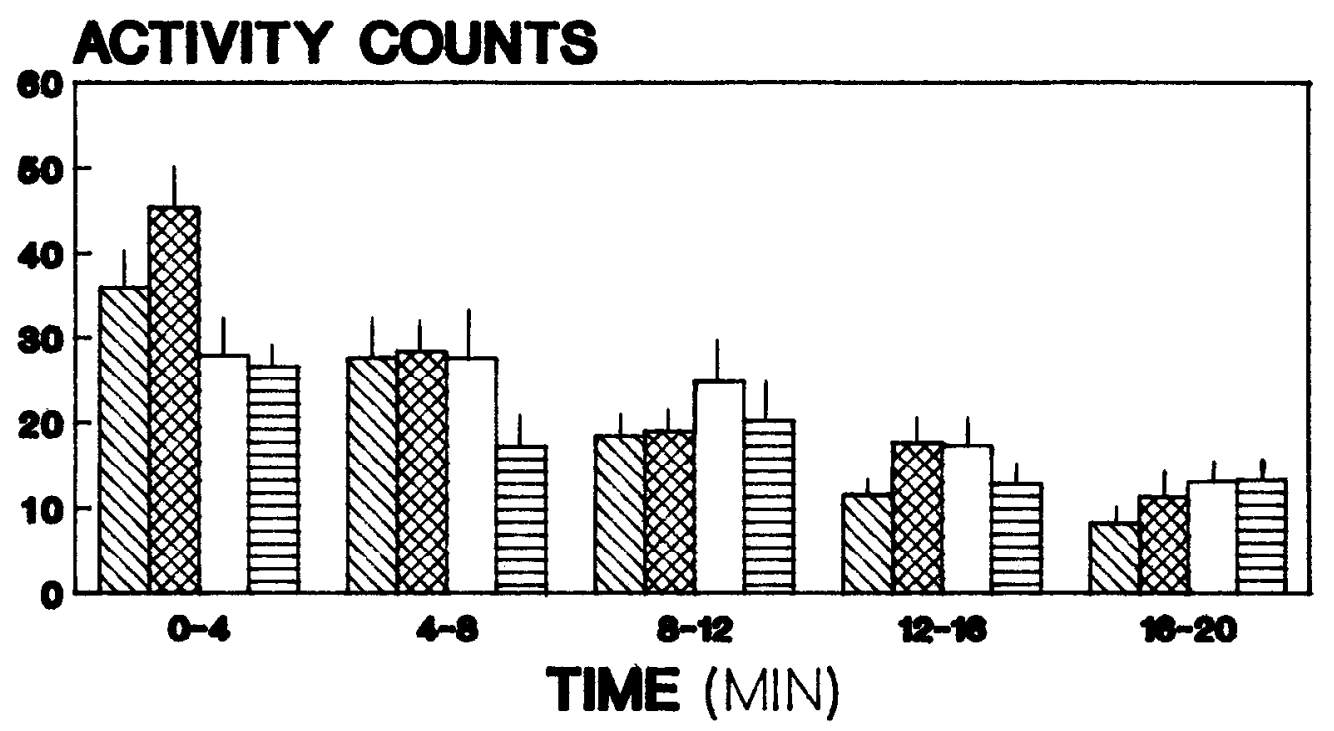

TREATMENT 

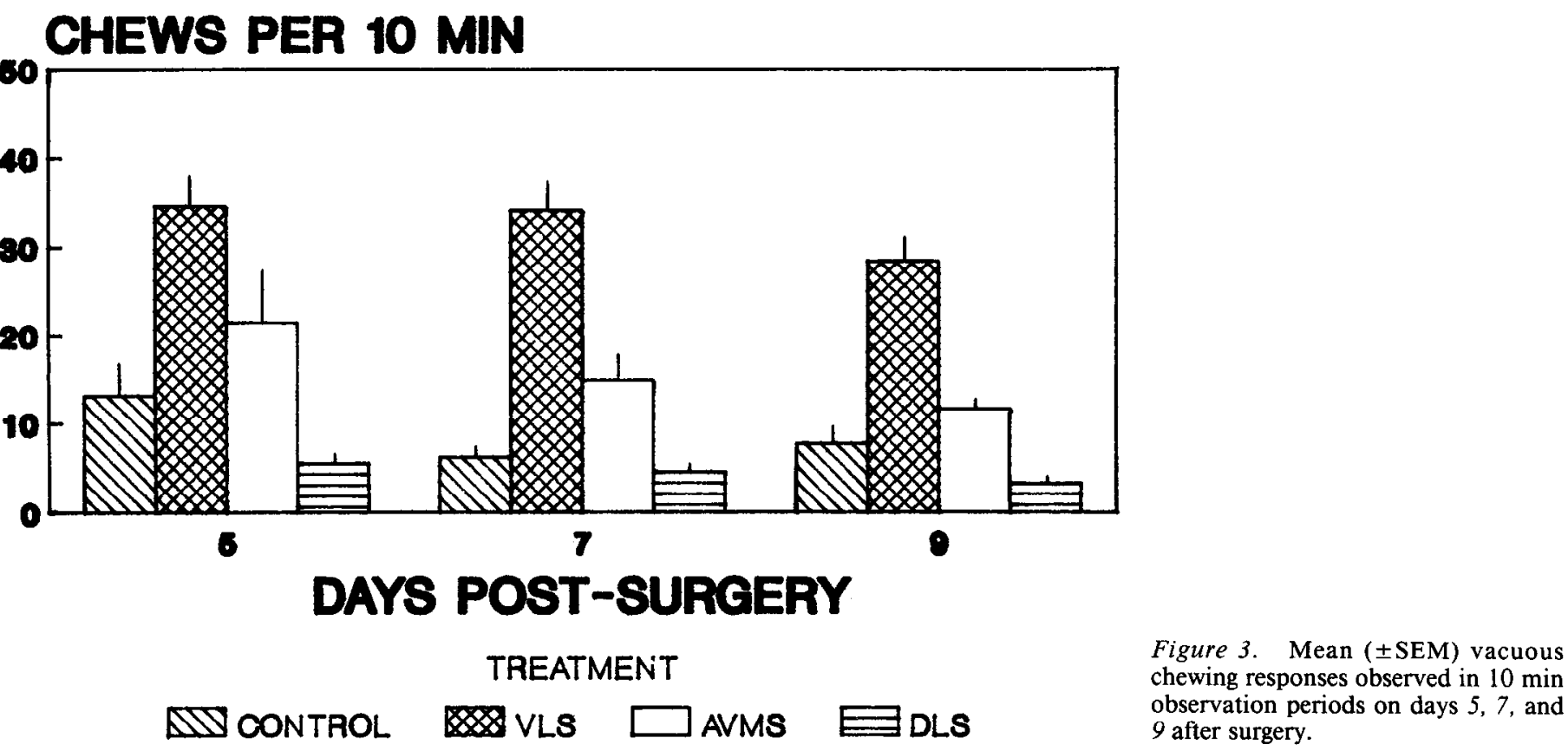

tion and HP administration produced a pronounced facial tremor in addition to high levels of vacuous chewing.

Rearing was suppressed in all three experimental groups after HP administration. Mean ( \pm SEM) numbers of rears after vehicle or HP treatment were as follows: vehicle-control, 23.5 (3.6); VLS, 23.8 (3.8); AVMS, 18.4 (3.9); DLS, 11.7 (2.1); haloperidol-control, 3.0 (2.0); VLS, 3.6 (2.0); AVMS, 2.6 (1.0); DLS, $0.8(0.33)$. ANOVA indicated that while there was no significant difference between experimental groups $[F(3,32)=2.2$, NS $]$, HP administration significantly decreased rearing behaviors in all three experimental groups $(F(1,32)=101.7 ; p<0.001)$. There was no interaction effect between experimental group and HP administration $[F(3,32)=1.9$, NS]. Among all 27 rats that received injections of 6-OHDA, there was a significant positive correlation between rearing and vacuous chewing $(r=0.37, p$ $<0.05)$.

\section{HPLC assays}

Figure 6 shows approximate locations of 6-OHDA injection sites. Tissue assay data (Table 1 ) revcalcd that there was a significant effect of treatment on DA levels in the nucleus accumbens $[F(3,32)=6.36 ; p<0.01]$, and post hoc tests indicated that only rats with AVMS placements significantly differed from controls in this region. Levels of DA in AVMS were significantly affected by 6 -OHDA treatment $[F(3,32)=26.7 ; p<0.001]$, with the group that received AVMS injections of 6-OHDA dif-

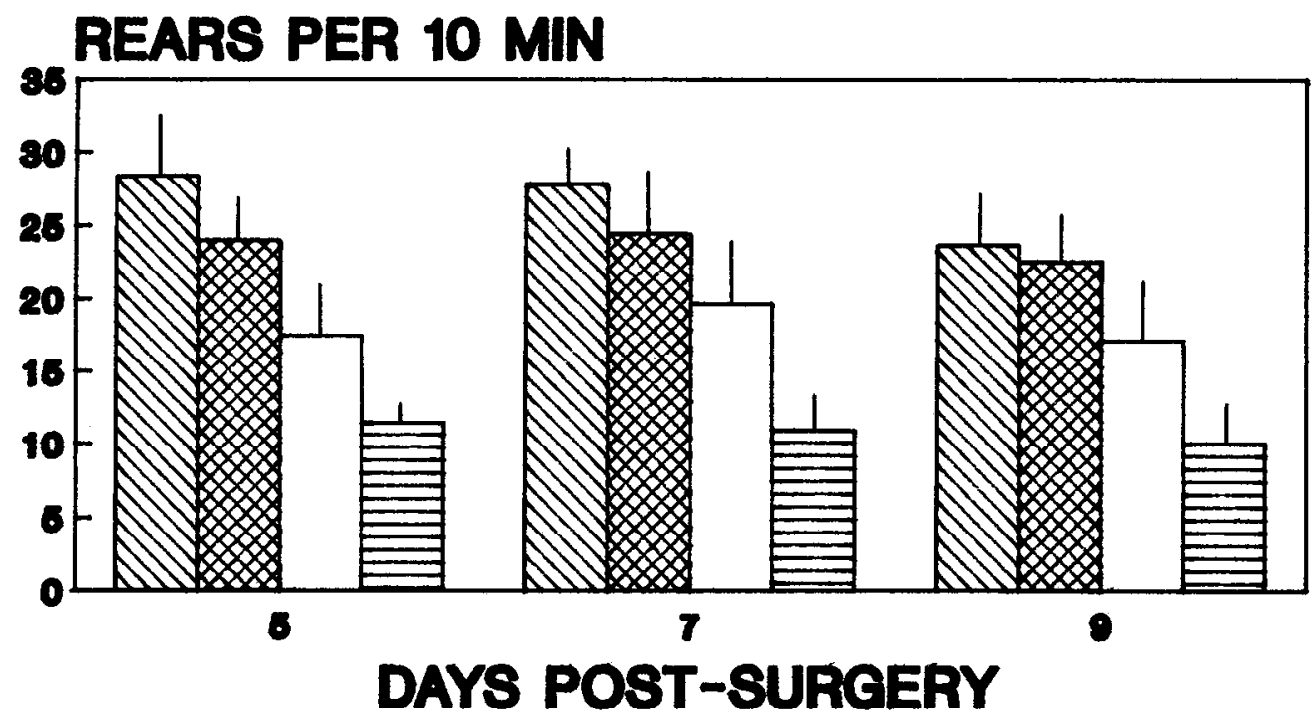

TREATMENT

SIV CONTROL

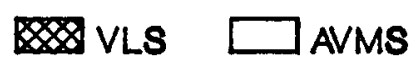

ELS
Figure 4. Mean ( $\pm \mathrm{SEM})$ rearing responses observed in 10 min periods on days 5,7 , and 9 after surgery. 
CHEWS PER 10 MIN

Figure 5. Mean $( \pm \mathrm{SEM})$ vacuous chewing responses observed in $10 \mathrm{~min}$ periods after administration of vehicle and $0.4 \mathrm{mg} / \mathrm{kg} \mathrm{HP}$.

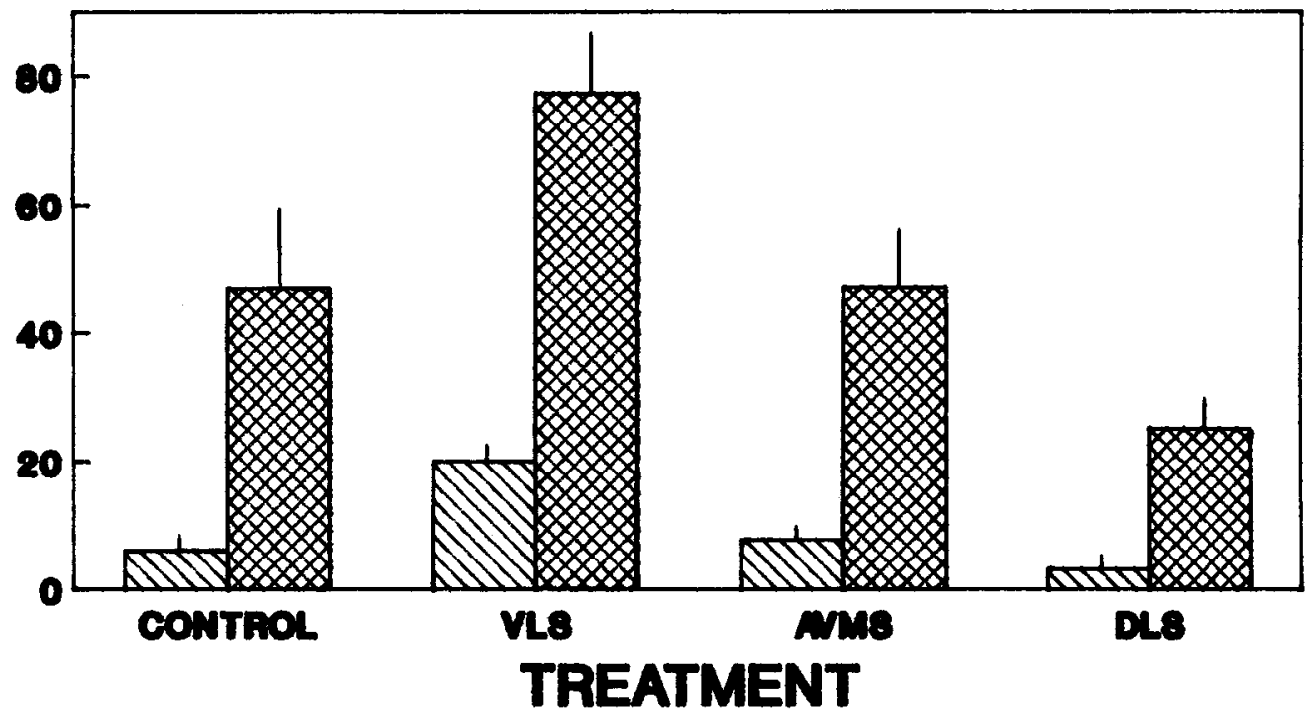

VEHICLE $0.4 \mathrm{mg} / \mathrm{kg} \mathrm{HP}$ fering from control rats. VLS DA levels were significantly affected by 6 -OHDA treatment $[F(3,32)=13.6 ; p<0.001]$ only in rats with VLS 6-OHDA injection. In DLS, there was a significant overall effect of 6 -OHDA treatment $[F(3,32)=9.46 ; p$ $<0.01]$, and post hoc analyses revealed that all three 6-OHDA groups had significantly reduced DA levels in DLS.

\section{Correlational analyses}

Table 2 is a correlational matrix for several behavioral and neurochemical parameters in all 27 rats that had received injections of 6-OHDA. Among all DA-depleted rats, DA levels in VLS were positively correlated with food intake and negatively correlated with vacuous chewing. In addition, food intake and vacuous chewing were inversely correlated with each other. Within the group of nine rats that had received VLS injections of 6-OHDA, there was a significant correlation between food intake and chewing $(r=-0.83 ; p<0.01)$, a significant correlation between DA levels in VLS and vacuous chewing $(r=$ $-0.75 ; p<0.01$ ), and a significant correlation between DA levels in VLS and food intake $(r=0.70 ; p<0.01)$. Although rats with VLS injections of 6-OHDA had dopamine depletions in DLS as well as VLS, there was neither a significant correlation between DLS DA and vacuous chewing in this group $(r=-0.41$, NS), nor a significant partial correlation between DLS DA levels and chewing when the analysis controlled for VLS DA levels ( $r$ $=-0,49$, NS).

\section{Discussion}

Depletion of DA in the VLS caused pronounced motor impairments. These rats had deficits in daily food consumption and showed significant increases in vacuous chewing responses. Injections of 6-OHDA into the AVMS and the DLS did not induce either of these effects. The only significant effect of DLS injections of 6-OHDA was to decrease rearing behavior. These data support the notion that the neostriatum of the rat is functionally heterogeneous and that DA in the VLS is important for oral motor control. Also, these results demonstrate that deple- tion of DA in VLS, as well as impairing normal motor function, can induce vacuous chewing movements.

Although the rats with VLS DA depletion showed increases in vacuous chewing and impairments in feeding, these rats were not akinetic. They showed no deficits in spontaneous locomotor activity, and in fact were more active than control rats in the early part of the activity test session. Rats with VLS DA depletion also had no significant reduction in rearing behavior, and DA levels in VLS were inversely correlated with rearing (Table 2). Among all DA-depleted rats, rearing was positively correlated with vacuous chewing on days 5,7 , and 9 (Table 2) and also after HP injection. These data indicate that the induction of vacuous chewing in rats with VLS DA depletions is not an artifact that is produced by a general decrease in movement (Levy et al., 1987). Also, the present results indicate that akinesia is dissociable from impairments in feeding, because rats with VLS DA depletion had deficits in feeding but not locomotion or rearing. In the present study, there was no site at which 6-OHDA injection produced a significant decrease in locomotor activity. It has been demonstrated that large depletions of DA in nucleus accumbens can produce moderate but statistically significant reductions in spontaneous locomotor activity (Koob et al., 1978, 1981). It is possible that there is no specific locus at which DA depletion produces near-total loss of spontaneous activity and that the akinesia that is observed after extensive forebrain DA depletion (e.g., Zigmond and Stricker, 1973, 1984; Marshall et al., 1976; Schallert et al., 1978) is related to the widespread nature of the DA depletions in those studies.

Although rats with VLS DA depletion showed reductions in food intake, these rats were not totally aphagic. Observations of these rats indicated that these animals were attempting to eat but were inefficient in their feeding behavior. These findings are consistent with the report by Salamone et al. (1990c) that rats recovering from extensive forebrain DA depletion showed deficits in feeding efficiency that were correlated with DA depletion in the lateral, but not the medial, striatum. Recent work from our laboratory indicated that rats with VLS DA depletions did 


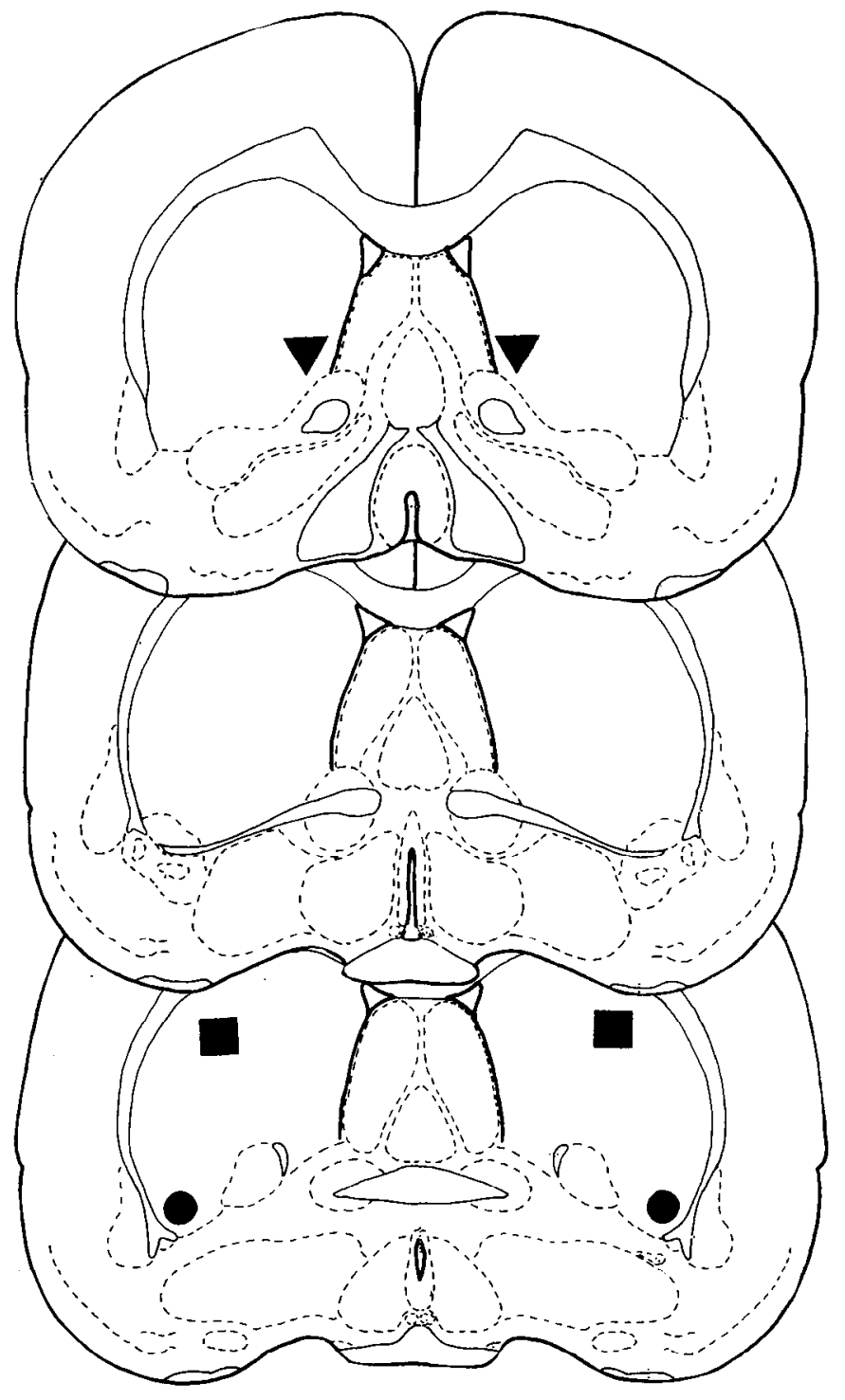

Figure 6. Approximate locations of injection placements in AVMS (triangles), VLS (circles), and DLS (squares).

not have a deficit in time spent feeding but did have deficits in the local rate of food intake, and problems with oral and forepaw usage during feeding (Rogers, 1991). In the present study, most rats with VLS DA depletions recovered food intake within a week after surgery, and all rats could maintain themselves on dry food by day 20 after surgery. These results are consistent with previous studies of recovery of function after near-total depletion of forebrain DA (Zigmond and Stricker, 1973; Stricker and Zigmond, 1976; Zigmond et al., 1984; Salamone et al., 1990c). However, compared to rats with extensive DA depletions, rats with VLS DA depletions in the present study showed a feeding deficit that was relatively less severe, and a recovery of feeding that was somewhat more rapid. These differences may be related both to the magnitude and the extent of the DA depletions produced by local VLS injection of 6-OHDA.

The functional heterogeneity of the neostriatum reflects the different inputs and outputs of each subregion. The AVMS placement used in the present studies overlaps with the striatal regions that receive input from medial and sulcal prefrontal

\begin{tabular}{|c|c|c|c|c|}
\hline \multirow{2}{*}{$\begin{array}{l}\text { Brain } \\
\text { region }\end{array}$} & \multirow[b]{2}{*}{ Control } & \multicolumn{3}{|c|}{ Treatment group } \\
\hline & & AVMS & VLS & DLS \\
\hline \multicolumn{5}{|c|}{ Nucleus accumbens } \\
\hline Mean & 7.71 & $4.99^{*}$ & 7.08 & 7.92 \\
\hline SEM & 0.33 & 0.41 & 0.70 & 0.59 \\
\hline \multicolumn{5}{|l|}{ AVMS } \\
\hline Mean & 9.82 & $1.73^{*}$ & 7.91 & 9.41 \\
\hline SEM & 0.88 & 0.70 & 0.52 & 0.75 \\
\hline \multicolumn{5}{|l|}{ VLS } \\
\hline Mean & 9.24 & 6.63 & $2.24^{*}$ & 10.45 \\
\hline SEM & 1.51 & 0.82 & 0.67 & 0.69 \\
\hline \multicolumn{5}{|l|}{ DLS } \\
\hline Mean & 9.96 & $4.91^{*}$ & $4.13^{*}$ & $4.07^{*}$ \\
\hline SEM & 0.99 & 0.85 & 0.86 & 0.95 \\
\hline
\end{tabular}

cortex in the rat (Leonard, 1969; Beckstead, 1979; Divac and Diemer, 1980). In contrast, the lateral striatum receives input from motor and sensory cortices (Webster, 1961; Wise and Jones, 1977; Collins, 1978). Considerable evidence indicates that the lateral striatum of rats and the putamen of primates are organized in a somatotopic manner (Alexander and Delong, 1985; Pisa and Schranz, 1988). In primates, there is a ventral-dorsal gradient, with orofacial and forelimb motor control being localized in more ventral areas, and trunk and hindlimb control in more dorsal regions. In our studies of rats, the rearing deficit produced by DLS injections of 6-OHDA is consistent with the hypothesis that the DLS is involved in control of trunk and hindlimb motor control. The VLS of the rat is involved in motor control of oral (Kelley et al., 1988; Pisa, 1988a) and forepaw (Evenden and Robbins, 1984; Sabol et al., 1985; Whishaw et al., 1986; Pisa, 1988a) regions, both of which are important for food handling and consumption. Also, vacuous chewing responses in rats are related to neurochemical processes in the VLS. The VLS is the only subregion of striatum in which vacuous chewing is produced by physostigmine (Kelley et al., 1989) or pilocarpine (Salamone et al., 1990a). In the present study, correlational analyses indicated that DA depletion in the VLS was closely related to the production of vacuous chewing but that DA depletion in AVMS and DLS was not.

Table 2. Correlation coefficients between behavioral and neurochemical parameters in all 27 rats that received 6-OHDA injections

\begin{tabular}{lllllll} 
& VLS & DLS & & & & \\
& DA & DA & Chewing & Rearing & Feeding & Activity \\
\hline AVMS DA & 0.12 & 0.17 & -0.11 & -0.12 & -0.18 & -0.13 \\
VLS DA & - & 0.23 & $-0.88^{*}$ & $-0.46^{*}$ & $0.72^{*}$ & -0.29 \\
DLS DA & - & - & -0.19 & -0.02 & 0.22 & 0.09 \\
Chewing & - & - & - & $0.53^{*}$ & $-0.70^{*}$ & 0.26 \\
Rearing & - & - & - & - & -0.24 & $0.56^{*}$ \\
Feeding & - & - & - & - & - & -0.07 \\
\hline
\end{tabular}

${ }^{*}, p<0.05$. 
Because vacuous chewing responses can be produced by chronic administration of neuroleptics (Iversen et al., 1980; Ellison et al., 1987; Stoessl et al., 1989), it could be argued that these responses are related to the development of DA receptor supersensitivity and could in fact represent a hyperactivity of DA transmission due to DA release onto these additional receptors. If DA release onto super-sensitive DA receptors was producing vacuous chewing in DA-depleted rats, then HP should have actually decreased vacuous chewing. In fact, blockade of DA receptors or depletion of VLS DA both increased vacuous chewing, and administration of HP to rats with VLS DA depletion resulted in still higher levels of chewing responses. These data are consistent with previous reports that intraventricular administration of 6-OHDA facilitated the vacuous chewing produced by chronic HP (Gunne et al., 1982). Although it is possible that vacuous chewing can result from several different conditions, the present rcsults indicate that reduced functional DA activity is associated with vacuous chewing.

Several lines of evidence suggest that vacuous chewing in rats shares some characteristics with human parkinsonian symptoms. Although parkinsonian tremor in humans usually involves the hand, there also are reports of up-and-down jaw movements, and movements that resemble mastication, in idiopathic and neuroleptic-induced parkinsonism (Sovner and Dimascio, 1977; Adams and Victor, 1981; Yassa and Lal, 1986). The involvement of $\mathrm{DA}$ and $\mathrm{ACh}$ systems, as well as the interaction between these transmitters, is similar in human parkinsonism and vacuous chewing in rats. For example, vacuous chewing responses are produced or exacerbated by decreasing striatal DA levels (Gunne et al., 1982; Salamone et al., 1990b; see also above), by acute or subchronic administration of DA antagonists (Glassman and Glassman, 1980; Rupniak et al., 1985; Salamone et al., 1990b), or by administration of cholinomimetic drugs (Rupniak et al., 1983, 1985; Salamone et al., 1986, 1990a,b; Kelley et al., 1989). Anticholinergic drugs, which are used to treat neuroleptic-induced parkinsonism (Duvoisin, 1967; Marsden et al., 1975; McEvoy, 1983), have been shown to reduce vacuous chewing induced by DA antagonists (Rupniak et al., 1983) and cholinomimetics (Rupniak et al., 1983; Salamone et al., 1986, 1990a; Stewart et al., 1989). Vacuous chewing produced by pilocarpine was reduced by the DA agonist apomorphine (Stewart et al., 1988). In the present experiment, administration of HP to rats with VLS DA depletion caused substantial increases in vacuous chewing and also induced facial tremors and paw shaking in some rats.

Vacuous chewing movements in rats involve a rapid oscillation of the lower jaw and have some of the characteristics of an oral tremor. According to Findley and Gresty (1981), a tremor is defined as an involuntary periodic oscillation of a body member. It is possible that vacuous chewing movements in rats could be used to study how interference with DA systems leads to the development of tremulous movements. Continued research is being used to provide a further pharmacological, anatomical, and behavioral characterization of vacuous chewing in rats. It will be important to determine if vacuous chewing responses are reduced by L-dopa or by thalamic lesions. In addition, vacuous chewing may provide a model system for studying the cellular neurophysiology of tremorogenic conditions. Studies are underway that utilize electromyographic recording of jaw muscles, in order to characterize the frequency and pattern of muscle activities shown during these movements.

\section{References}

Adams RD, Victor M (1981) Tremors, myoclonus, spasms and tics. In: Principles of neurology, pp 69-79. New York: McGraw-Hill.

Alexander GE, Delong MR (1985) Microstimulation of the primate neostriatum. 2. Somatotopic organization of striatal microexcitable zones and their relation to neuronal response properties. J Neurophysiol 53:1417-1430.

Beckstead RM (1979) An autoradiographic examination of cortical and subcortical projections of the mediodorsal projection (prefrontal) cortex in the rat. J Comp Neurol 184:43-62.

Buonamici M, Maj R, Pagani F, Rossi AC, Khazan N (1986) Tremor at rest in unilaterally 6-OHDA-induced substantia nigra lesioned rats. Neuropharmacology 25:323-325.

Collins RC (1978) Kindling of neuroanatomic pathways during recurrent focal penicillin seizures. Brain Res 150:503-517.

Divac I, Diemer NH (1980) Prefrontal system in the rat visualized by means of labeled deoxyglucose - further evidence of functional heterogeneity of the neostriatum. J Comp Neurol 190:1-13.

Dunnett SB, Iversen SD (1982) Sensorimotor impairments following localized kainic acid and 6-hydroxydopamine lesions of the neostriatum. Brain Res 248:121-127.

Duvoisin RC (1967) Cholinergic-anticholinergic antagonism in parkinsonism. Arch Neurol 17:124-136.

Ellison G, See RE (1989) Rats administered chronic neuroleptics develop oral movements which are similar in form to those in humuns with tardive dyskinesia. Psychopharmacology 98:564-566.

Ellison G, See R, Levin E, Kinney J (1987) Tremorous mouth movements in rats administered chronic neuroleptics. Psychopharmacology 92:122-126.

Evenden JL, Robbins TW (1984) Effects of unilateral 6-hydroxydopamine lesions of the caudate-putamen on skilled forelimb use in the rat. Behav Brain Res 14:61-68.

Fairley PC, Marshall JF (1987) Dopamine in the lateral caudate-putamen of the rat is essential for somatosensory orientation. Behav Neurosci 100:652-663.

Findley LJ, Gresty MA (1981) Tremor. Br J Hosp Med 26:16-32.

Glassman RB, Glassman HN (1980) Oral dyskinesia in brain-damaged rats withdrawn from neuroleptic: implication for models of tardive dyskinesia. Psychopharmacology 69:19-25.

Gunne LM, Growden J, Glaeser B (1982) Oral dyskinesia in rats following brain lesions and neuroleptic administration. Psychopharmacology 77:134-139.

Harbaugh RE, Roberts DW, Coombs DW, Saunders RL, Reeder TM (1984) Preliminary report: intracranial cholinergic drug infusion in patients with Alzheimer's disease. Neurosurgery 15:514-518.

Iversen SD, Howells RB, Hughs RP (1980) Behavioral consequences of long-term treatment with neuroleptic drugs. Adv Biochem Psychopharmacology 24:305-313.

Johansson P, Casey DE, Gunne LM (1986) Dose-dependent increases in rat spontaneous chewing rates during long-term administration of haloperidol but not clozapine. Psychopharmacology Bull 22:10171019.

Keefe KA, Salamone JD, Zigmond MJ, Stricker EM (1989) Paradoxical kinesia in parkinsonism is not caused by dopamine release: studies in an animal model. Arch Neurol 46:1070-1075.

Kelley AE, Lang CG, Gauthier AM (1988) Induction of oral stereotypy following amphetamine microinjection into a discrete subregion of the striatum. Psychopharmacology 95:556-559.

Kelley AE, Bakshi VP, Delfs JM, Lang CG (1989) Cholinergic stimulation of the ventrolateral striatum elicits mouth movements in rats: pharmacological and regional specificity. Psychopharmacology 99: 542-549.

Koob GF, Riley SJ, Smith SC, Robbins TW (1978) Effects of 6-hydroxy-dopamine lesions of the nucleus accumbens septi and olfactory tubercle on feeding, locomotor activity and amphetamine anorexia in the rat. J Comp Physiol Psychol 92:917-927.

Koob GF, Stinus L, Le Moal M (1981) Hyperactivity and hypoactivity produced by lesions to the mesolimbic dopamine system. Behav Brain Res 3:341-359.

Leonard CM (1969) The prefrontal cortex of the rat. I. Cortical projection of the medial dorsal nucleus. II. Efferent connections. Brain Res 12:321-343. 
Levy AD, See RE, Levin ED, Ellison GD (1987) Neuroleptic-induced oral movements in rats: methodological issues. Life Sci 41:14991506.

Marsden CD, Tarsy D, Baldessarini RJ (1975) Spontaneous and druginduced movement disorders in psychotic patients. In: Psychiatric aspects of neurological disease (Bendon DF, Blumer D, eds), pp 216266. New York: Grune \& Stratten.

Marshall JF, Richardson JS, Teitelbaum P (1974) Nigrostriatal bundle damage and the lateral hypothalamic syndrome. J Comp Physiol Psychol 87:808-830.

Marshall JF, Levitan D, Sticker EM (1976) Activation-induced restoration of sensorimotor functions in rats with dopamine-depleting brain lesions. J Comp Physiol Psychol 87:808-830.

McEvoy JP (1983) The clinical use of anticholinergic drugs as treatment for extrapyramidal side effects of neuroleptic drugs. J Clin Psychopharmacology 3:288-301.

Noring U, Povlesen UJ, Casey DE, Gerlach J (1984) Effect of a cholinomimetic drug (RS 86) in tardive dyskinesia and drug-related parkinsonism. Psychopharmacology 84:569-571.

Pisa M (1988a) Motor somatotopy in the striatum of rat: manipulation, biting and gait. Behav Brain Res 27:21-35.

Pisa M (1988b) Motor functions of the striatum in the rat: critical role of the lateral region in tongue and forelimb reaching. Neuroscience 24:453-463.

Pisa M, Schranz JA (1988) Dissociable motor roles of the rat's striatum conform to somatotopic model. Behav Neurosci 102:429-440.

Rogers S (1991) Effect of dopamine depletion in nucleus accumbens and striatum on feeding behavior in rats. Unpublished honors thesis, University of Connecticut.

Rupniak NMJ, Jenner P, Marsden CD (1983) Cholinergic modulation of perioral behavior induced by chronic neuroleptic administration to rats. Psychopharmacology 79:226-230.

Rupniak NMJ, Jenner P, Marsden CD (1985) Pharmacological characterization of spontaneous or drug-induced purposeless chewing movements in rats. Psychopharmacology 85:71-79.

Rupniak NMJ, Jenner P, Marsden CD (1986) Acute dystonia induced by neuroleptic drugs. Psychopharmacology 88:403-419.

Sabol KE, Neill DB, Wages SA, Church WH, Justice JB (1985) Dopamine depletion in a striatal subregion disrupts performance of a skilled motor task in the rat. Brain Res 335:33-43.

Salamone JD, Lalies MD, Channell SL, Iversen SD (1986) Behavioral and pharmacological characterization of the mouth movements induced by muscarinic agonists in the rat. Psychopharmacology 88: $467-471$.

Salamone JD, Johnson J, McCullough LD, Steinpreis RE (1990a) Lateral striatal cholinergic mechanisms involved in oral motor activities in the rat. Psychopharmacology 102:529-534.

Salamone JD, Steinpreis RE, Taylor ME, Jicha G (1990b) Tremulous chewing movements in rats induced by haloperidol, pilocarpine and striatal dopamine depletion. Soc Neurosci Abstr 16:132.

Salamone JD, Zigmond MJ, Stricker EM (1990c) Characterization of the impaired feeding behavior in rats given haloperidol or dopaminedepleting brain lesions. Neuroscience 39:17-24.

Schallert T, Whishaw IQ, Ramirez VD, Teitelbaum P (1978) Compulsive, abnormal walking caused by anticholinergics in akinetic, 6-hydroxydopamine-treated rats. Science 199:1461-1463.
Schwab RS, Zieper I (1965) Effects of mood, motivation, stress and alertness on the performance in Parkinson's disease. Psychiatr Neurol 150:345-357.

See RE, Levin ED, Ellison GD (1988) Characteristics of oral movements in rats during and after chronic haloperidol and fluphenazine administration. Psychopharmacology 94:421-427.

Sovner R, Dimascio A (1977) The effect of benztropine mesylate in the rabbit syndrome and tardive dyskinesia. Am J Psychiatr 134: 1301-1302.

Stewart BR, Jenner P, Marsden CD (1988) Pharmacological characterization of pilocarpine-induced chewing in the rat. Psychopharmacology 96:55-62.

Stewart BR, Jenner P, Marsden CD (1989) Assessment of the muscarinic receptor subtype involved in the mediation of pilocarpineinduced chewing behavior. Psychopharmacology 97:228-234.

Stoessl AJ, Dourish CT, Iversen SD (1989) Chronic neuroleptic-induced mouth movements in rats: suppression by CCK and selective dopamine $D_{1}$ and $D_{2}$ reccptor antagonists. Psychopharmacology 98 : 372-379.

Stricker EM, Zigmond MJ (1976) Recovery of function following damage to central catecholamine-containing neurons: a neurochemical model for the lateral hypothalamic syndrome. In: Progress in psychobiology and physiological psychology (Sprague JM, Epstein AN, eds). New York: Academic.

Ungerstedt $U$ (1971) Aphagia and adipsia after 6-hydroxydopamine induced degeneration of the nigro-striatal dopamine system. Acta Physiol Scand 82[Suppl 367]:95-122.

Uretsky NJ, Schoenfeld RI (1971) Effect of L-DOPA on the locomotor activity of rats pretreated with 6-hydroxydopamine. Nature 234:157159

Waddington JL (1990) Spontaneous orofacial movements induced in rodents by very long-term neuroleptic drug administration: phenomenology, pathophysiology and putative relationship to tardive dyskinesia. Psychopharmacology 101:431-447.

Waddington JL, Molloy AG (1987) The status of late-onset vacuous chewing/perioral movements during long-term neuroleptic treatment in rodents: tardive dyskinesia or dystonia. Psychopharmacology 91: 136-137.

Webster KE (1961) Cortico-striate interrelations in the albino rat. J Anat 95:523-543.

Whishaw IQ, O'Connor WT, Dunnet SB (1986) The contribution of motor cortex, nigrostriatal dopamine and caudate-putamen to skilled forepaw use in the rat. Brain 109:805-843.

Wise SP, Jones EG (1977) Cells of origin and terminal distribution of descending projections of the rat somatic sensory cortex. J Comp Ncurol 175:129-158.

Yassa R, Lal S (1986) Prevalence of the rabbit syndrome. Am J Psychiatr 143:656-657.

Zigmond MJ, Stricker EM (1973) Recovery of feeding and drinking by rats after intraventricular 6-hydroxydopamine or later hypothalamic lesions. Science 182:717-720.

Zigmond MJ, Stricker EM (1984) Parkinson's disease: studies with an animal model. Life Sci 35:5-18.

Zigmond MJ, Acheson AL, Stachowiak MK, Stricker EM (1984) Neurochemical compensation after nigrostriatal bundle injury in an animal model of preclinical parkinsonism. Arch Neurol 41:856-861. 